\title{
Nature of branon dark matter
}

\author{
A. L. Maroto \\ Departamento de Física Teórica, Universidad Complutense de Madrid, 28040 Madrid, Spain
}

(Received 29 October 2003; published 26 February 2004)

\begin{abstract}
We explore the different possibilities for branons as dark matter candidates. We consider a general braneworld model, parametrized by the number of extra dimensions, $N$, the fundamental scale of gravity $M_{D}$, the brane tension scale $f$, and the branon mass $M$. We analyze the parameter region in which branons behave as collisionless thermal relics, either cold or hot (warm), together with less standard scenarios in which they are strongly self-interacting or produced non-thermally.
\end{abstract}

DOI: 10.1103/PhysRevD.69.043509

PACS number(s): 95.35.+d, 11.10.Kk, 11.25.-w

\section{INTRODUCTION}

Cold dark matter (CDM) is a fundamental ingredient of the current cosmological standard model. An enormous variety of observations at very large scales $(\gtrsim 1 \mathrm{Mpc})$, from cosmic microwave background anisotropies, galaxy surveys, cluster abundances to the Ly- $\alpha$ forest are successfully explained within this framework. However, to achieve these goals, the dark matter component is required to behave as a collisionless nonrelativistic particle fluid. This poses an important problem from the particle physics point of view. Indeed, no candidate with the assumed properties exists within the known particles, and therefore physics beyond the standard model (SM) appears as the only feasible solution. Among the proposed candidates, we find, on one hand, the axion, which is the Goldstone boson associated with the spontaneous breaking of the Peccei-Quinn symmetry postulated to solve the strong $C P$ problem of $\mathrm{QCD}$. The production of axions in the early universe mainly takes place through the so called misalignment mechanism in which the $\Theta$ angle is initially displaced from its equilibrium value $\Theta$ $=0$ and oscillates coherently. Such oscillations can be intrepreted as a zero-momentum Bose-Einstein condensate which essentially behaves as a non-relativistic matter fluid. Despite the fact that axions are light particles, this nonthermal mechanism produces cosmologically important energy densities. On the other hand we have the thermal relics, produced by the well-known freeze-out mechanism in an expanding universe. They are typically weakly interacting massive particles (WIMPs) such as the neutralino in supersymmetric theories [1] (for a recent review see also [2]). In addition to their weak interactions with SM particles, these candidates usually also have very weak self-interactions (collisionless).

Despite the success of CDM at large scales, the model exhibits certain difficulties at sub-galactic scales. In particular, high resolution $N$-body simulations of dark halos show cuspy density profiles $[3,4]$ which contradict observations from low surface brightness galaxies [5] and dwarfs [6,7] that suggest flat density profiles. In addition, CDM also predicts too many small subhalos within simulated larger systems [8,9], in contradiction with observations of the number of satellite galaxies in the local group. Solutions which aim to reduce the power at small scales, but keeping the good properties of CDM at large scales, have been proposed at both the astrophysical and a more fundamental level. They include modifications of the primordial power spectrum at small scales [10], or of the collapse histories of CDM and baryons [11], but also modifications in the nature of the dark matter. There are two main proposals along the latter lines, namely, warm dark matter (WDM) [12] and self-interacting dark matter (SDM) [13]. In the WDM scenario the particle dark matter is still collisionless, but much lighter with $M$ $\lesssim 1 \mathrm{keV}$. Free-streaming exponentially suppresses the power spectrum below the scale $\lambda_{F S} \simeq 0.2\left(\Omega_{B r} h^{2}\right)^{1 / 3}$ $\times(\mathrm{keV} / M)^{4 / 3} \mathrm{Mpc}$. This suppression solves the sub-halo problem provided $M \simeq 1 \mathrm{keV}$ [14]; however the central cusp problem requires a much lighter particle $M \leqslant 300 \mathrm{eV}$ [14]. In addition, the Ly- $\alpha$ forest in quasar spectra observations puts a severe lower bound on the mass of the WDM candidate, namely, $M \gtrsim 750 \mathrm{eV}$, which makes the simplest models infeasible [15]. A second possibility is to take into account dark matter self-interactions. Thus, if the mean free path of dark matter particles is of the order of $\sim 1 \mathrm{kpc}$, but still with small annihilation cross sections, then heat can be transported out of the center of the halo smoothing out the central cusp, which now would become a more spherical core. In order for this mechanism to work, the total elastic dark matter cross section should be in the range [16]

$$
\frac{\sigma}{M} \sim 0.5-5 \mathrm{~cm}^{2} \mathrm{~g}^{-1}
$$

which is comparable to that of a nucleon-nucleon interaction.

However, the SDM model is also very strongly constrained by different observations. Thus, in order to avoid evaporation of halos on a Hubble time scale, the cross section should be sufficiently small $\sigma / M<0.3-1 \mathrm{~cm}^{2} \mathrm{~g}^{-1}$ [17]. In addition Chandra observations of the mass density profile of a certain cluster imposes a very strong limit $\sigma / M$ $<0.1 \mathrm{~cm}^{2} \mathrm{~g}^{-1}$ [18]. Finally, the elliptical shape of a particular cluster determined by lensing data leads to a even stronger limit $\sigma / M<0.02 \mathrm{~cm}^{2} \mathrm{~g}^{-1}$ [19], otherwise selfinteractions would erase ellipticity. The interpretation of these results together with other direct constraints can be found in [20]. It is also interesting to point out that the combination of both scenarios, i.e. self-interacting warm dark matter, could alleviate their individual problems [21]. Apart 
from SDM and WDM, an interesting proposal in [22] shows that non-thermal WIMP production could also help resolving the mentioned discrepancies.

The new requirements imposed by these models on the dark matter particles make it even more difficult to find appropriate candidates in the mentioned SM extensions. For that reason it is worth analyzing the potential of branon dark matter [23] as an alternative to the more common neutralino or axion cases. In this paper we study the different possibilities in terms of the brane-world parameters. We will consider the case in which branons and SM particles are the only relevant degrees of freedom at low energies. This happens whenever the brane tension scale $f$ is much smaller than the fundamental scale of gravity in $D=4+N$ dimensions $M_{D}$ [24].

The paper is organized as follows: after introducing branon dark matter and its interactions, we study its collisional (collisionless) nature essentially in terms of $M_{D}$ and $f$. Then we analyze the possibility of producing branons nonthermally, in a similar way to the misalignment mechanism, where now the brane coherently oscillates along the extra dimensions. We end with some conclusions.

\section{BRANON INTERACTIONS}

In the case in which translational invariance is an exact symmetry in the extra dimensions, the presence of the brane can break it spontaneously, and branons can be identified with the corresponding Goldstone bosons [25,26]. For the sake of definiteness, we will consider a particular model with three compact extra dimensions, although we expect that the results will depend only on the different energy scales involved in the problem and not on the details of the model. We will choose an extra space $B=S^{3}$, so that its isometry group $G(B)=S U(2) \times S U(2)$ is spontaneously broken down to $S U(2)$. Therefore, the coset space is $K=S U(2)$ $\times S U(2) / S U(2)=S U(2) \sim S^{3}=B$. We thus have three branons, and the coset space metric is nothing but the 3-sphere metric: $h_{\alpha \beta}(\pi)=\delta_{\alpha \beta}+\pi^{\alpha} \pi^{\beta} /\left(v^{2}-\pi^{2}\right)$, where $v=f^{2} R_{B}$ with $R_{B}=M_{D}^{-1}\left(M_{P} / M_{D}\right)^{2 / N}$ the size of the extra space $B$ and $M_{P}$ the usual Planck mass. Therefore, $v$ is the spontaneous symmetry breaking scale which sets the size of the coset space $K$.

As shown in [26], the low-energy branon dynamics is given, to lowest order in derivatives, by the non-linear sigma model corresponding to that symmetry breaking pattern, i.e.,

$$
\begin{aligned}
\mathcal{L}_{B r}= & \frac{1}{2} g^{\mu \nu} h_{\alpha \beta}(\pi) \partial_{\mu} \pi^{\alpha} \partial_{\nu} \pi^{\beta}+\mathcal{O}\left(p^{4}\right) \\
\simeq & \frac{1}{2} g^{\mu \nu} \partial_{\mu} \pi^{\alpha} \partial_{\nu} \pi^{\alpha}+\frac{1}{2 v^{2}} g^{\mu \nu} \pi^{\alpha} \pi^{\beta} \partial_{\mu} \pi^{\alpha} \partial_{\nu} \pi^{\beta}+\mathcal{O}\left(\pi^{6}\right) \\
& +\mathcal{O}\left(p^{4}\right) .
\end{aligned}
$$

We see that $v$ sets the strength of branon self-interactions to lowest order in derivatives. Notice however that the $\mathcal{O}\left(p^{4}\right)$ terms are not suppressed by the $v$ scale, but by the $f$ scale [26].
So far we have only considered massless branons; however, translational invariance in the extra dimensions can be explicitly broken, and branons can acquire mass. In such a case, we should include in the Lagrangian the corresponding breaking terms. As shown in $[27,28]$, such terms will include an arbitrary number of even powers of branon fields, i.e.,

$$
\mathcal{L}_{\text {break }}=-\frac{M^{2}}{2} \pi^{2}+\frac{\lambda}{4 !} \frac{M^{2}}{v^{2}} \pi^{4}+\mathcal{O}\left(\pi^{6}\right) .
$$

Since branons are pseudoscalar particles, parity on the brane forbids terms with an odd number of fields [29]. Notice that here we are assuming for simplicity that the branons are degenerate, with a mass $M$ which is small compared to $v$. Therefore the explicit breaking terms can also be organized in powers of the branon mass, which is considered to be of the same order as a derivative in the power counting. Accordingly, we expect the parameter $\lambda$, which depends on the specific breaking mechanism, to be of order one. This is completely analogous to the pion dynamics in chiral perturbation theory [30], in which $v$ would play the role of the pion decay constant and the expression in Eq. (3) with $\lambda$ $=1$ corresponds to the expansion in $\pi$ fields of the lowest order mass term.

Branons also interact with the SM particles through their energy momentum tensor. Again the lowest order term in derivatives was obtained in $[26,28]$ :

$$
\mathcal{L}_{B r-S M}=\frac{1}{8 f^{4}}\left(4 \partial_{\mu} \pi^{\alpha} \partial_{\nu} \pi^{\alpha}-M^{2} \pi^{\alpha} \pi^{\alpha} g_{\mu \nu}+\ldots\right) T_{S M}^{\mu \nu}
$$

where the ellipsis stands for higher order terms in $\pi$ fields. We see that the branon-SM interactions are controlled by the brane tension scale $f$. What is important from the viewpoint of the dark matter problem is that branon self-interactions are controlled by a completely different energy scale from branon-SM interactions. This means that it is possible to have strong self-interactions and simultaneously small annihilation cross sections into SM particles, so that the branon relic abundance can be important. In addition, since branons always interact by pairs this implies that they are stable particles and therefore they satisfy all the requirements of a dark matter candidate.

\section{COLLISIONAL VS COLLISIONLESS BRANONS}

Branons were in thermal equilibrium with the rest of the SM particles when the temperature of the universe was above their decoupling temperature, which depends on $f$ and $M$ through the corresponding annihilation cross section. Assuming that the evolution of the universe was standard up to a temperature of the order of the brane tension scale, and that decoupling occurred below this temperature, which is indeed the case (see [23]), then the above effective Lagrangians can be used to compute the branon thermal relic abundances generated by the standard freeze-out mechanism. The calculation of the abundances [23,29] shows that there are two regimes in which the corresponding energy density could be compat- 
ible with the measurements of the cosmological dark matter, i.e. $\Omega_{B r} h^{2} \leq 0.129$ and also with LEP-II bounds [28]. On one hand we have a region in which $M \gtrsim 100 \mathrm{GeV}$ and $f \leqq M$, which corresponds to cold relics, and on the other, a region with $M \lesssim 180 \mathrm{eV}$ and $f \gtrsim 200 \mathrm{GeV}$ in which branons behave as hot or warm dark matter.

In this section we explore these two regimes and calculate the branon self-interactions in terms of the number of branon fields $N$ and the fundamental scale $M_{D}$. With that purpose we will obtain the branon-branon elastic cross section.

Since particles in the halo collide with very low relative velocities $v_{\text {rel }} \sim 10^{-3}$, the scattering amplitudes can be obtained from the above effective Lagrangian and they are given, for $\lambda=1$, by the well-known Weinberg low-energy theorem [30]

$$
A(s, t, u)=\frac{s-M^{2}}{v^{2}} .
$$

Assuming that the explicit breaking terms respect the remaining $S U(2)$ symmetry, branons transform as an $S U(2)$ triplet and it is possible to construct isospin $I=0,1,2$ amplitudes as

$$
\begin{aligned}
& T_{0}(s, t, u)=3 A(s, t, u)+A(t, s, u)+A(u, t, s), \\
& T_{1}(s, t, u)=A(t, s, u)-A(u, t, s), \\
& T_{2}(s, t, u)=A(t, s, u)+A(u, t, s),
\end{aligned}
$$

and also define the corresponding partial wave amplitudes with definite angular momentum $J$ :

$$
t_{I J}=\frac{1}{32 \pi} \int_{-1}^{1} d(\cos \theta) P_{J}(\cos \theta) T_{I}(s, t, u) .
$$

From the amplitude, the corresponding total elastic scattering cross section summed over final isospin states and averaged over initial ones in the non-relativistic limit reads

$$
\sigma_{t o t}=\frac{23 M^{2}}{384 \pi v^{4}}+\mathcal{O}\left(v_{r e l}^{2}\right)
$$

Apparently this cross section grows unboundedly with $M$; this is due to the fact that we are working with an effective theory, and tree-level unitarity violations are expected for sufficiently high values of $s \simeq 4 M^{2}$. In order to estimate the range of validity of the above result, we will use the wellknown unitarity limit

$$
\operatorname{Re} t_{I J} \leqslant \frac{1}{2 \rho}
$$

where $\rho=\left(1-4 M^{2} / s\right)^{1 / 2}$. From the dominant $t_{00}$ wave, we get in the mentioned limit

$$
\frac{M^{2}}{v^{2}} \leqslant \frac{16 \pi}{7 v_{r e l}}
$$

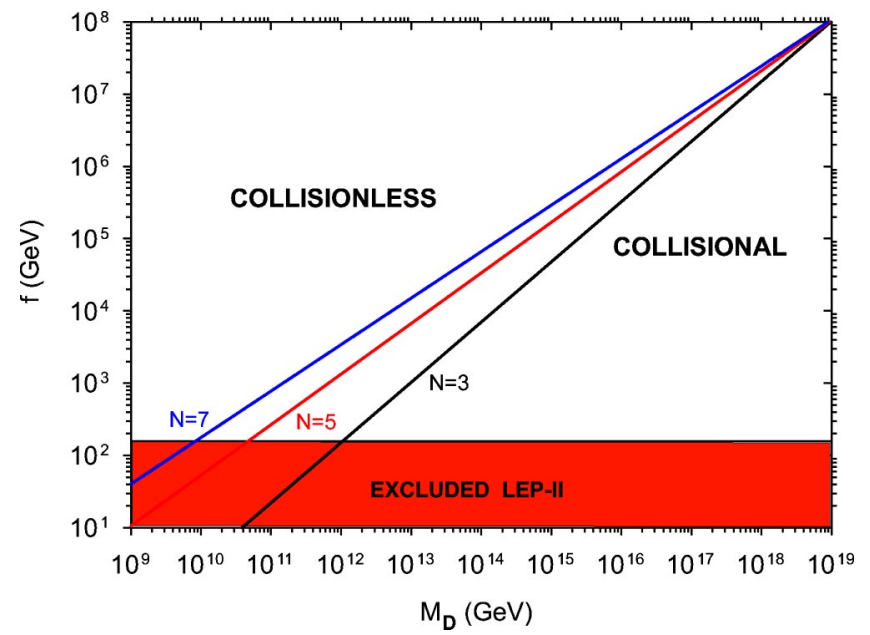

FIG. 1. Collisional vs collisionless regions in $f-M_{D}$ space for a model with $M=100 \mathrm{eV}$ and $N=3,5,7$. The dividing lines correspond to $\sigma_{\text {tot }} / M \simeq 0.5 \mathrm{~cm}^{2} \mathrm{~g}^{-1}$. The lower shaded area is excluded by LEP-II single photon analysis.

which is compatible with the general result derived in [31]. For typical values of $v_{r e l}$, we find $M / v \leq \mathcal{O}\left(10^{2}\right)$. Substituting back in Eq. (8), we find a limit for the cross section:

$$
\frac{\sigma_{t o t}}{M} \lesssim \frac{4 \times 10^{6}}{M^{3}} .
$$

In order for this kind of branons to be strongly selfinteracting they should satisfy $\sigma_{t o t} / M \geqslant 0.5 \mathrm{~cm}^{2} \mathrm{~g}^{-1}=2.3$ $\times 10^{3} \mathrm{GeV}^{-3}$, which implies $M \lesssim 12 \mathrm{GeV}$, in agreement with the general bound [31]. This immediately excludes cold branons as possible SDM candidates, according to the LEP-II limits mentioned before. Notice that, in any case, this is only a unitarity limit on the model; for larger masses, branon interactions could be sufficiently strong, but they will not be described by our effective theory.

Nevertheless, self-interacting warm branons with masses in the region $M \leqq 180 \mathrm{eV}$ are not excluded a priori by the previous bound. In principle, such a light particle would conflict with Ly- $\alpha$ forest observations, as commented before. However, such limits were derived assuming collisionless WDM. In the collisional case free-streaming is suppressed and the relevant cutoff scale for structure formation is the standard Jeans length $\lambda_{J}$, which can be substantially smaller than $\lambda_{F S}$ [14]. In any case a detailed calculation of the matter power spectrum would be needed for each particular model [21].

In Fig. 1 we have plotted the different regions in the $f-M_{D}$ plane for a branon mass $M \sim 100 \mathrm{eV}$. The curves are not very sensitive to the branon mass, since they scale as $M^{1 / 8}$. We see that for a given $f$, models become selfinteracting at sufficiently high $M_{D}$. Notice that the plotted regions are consistent with the assumption of a low-tension brane $f \ll M_{D}$. 


\section{THERMAL VS NON-THERMAL BRANONS}

So far we have considered branons as WIMPs which were produced thermally in the early universe. However, if the evolution of the universe was not standard before branon decoupling or the reheat temperature $T_{R H}$ was sufficiently low, branons were never in thermal equilibrium with radiation. However, they could still be produced non-thermally very much in the same way as axions [32]. Thus, if the maximum temperature reached in the universe was smaller than the freeze-out temperature $T_{R H} \ll T_{f}$, but larger compared to the explicit symmetry breaking scale $T_{R H} \gg \Lambda$ with $\Lambda=(M v)^{1 / 2}$, then brane fluctuations were initially essentially massless and decoupled from the rest of the matter fields. In this case, there is no reason to expect that the position in the extra dimension $Y_{0}$ at which the brane is created should coincide with the minimum of the branon potential $(Y=0)$. In general we expect $Y_{0} \sim \mathcal{O}\left(R_{B}\right)$, i.e. $\pi_{0} \sim v$ within a region of size $H^{-1}$ [32]. The evolution of the branon field is then simply that of a scalar field in an expanding universe. Thus, while $H \gg M$, the field remains frozen in its initial position $\pi=\pi_{0}$. Below the temperature $T_{i}$ for which $3 H\left(T_{i}\right) \simeq M$, the branon field oscillates around the minimum. These oscillations correspond to a zero-momentum branon condensate, its energy density behaving like nonrelativistic matter.

For the sake of simplicity we will consider the case of branons which are lighter than all the SM particles masses, i.e. we will assume that $M \ll 1 \mathrm{MeV}$ and that neutrinos are massless. In such a case the branon condensate cannot decay by particle production into massive SM particles, but still branons could annihilate through $2 \rightarrow 2$ processes into massless gauge bosons or neutrinos. Since the expectation value of $\pi$ varies on horizon scales, the typical momentum of branons can be estimated as $|\vec{p}| \sim H$, i.e. $|\vec{p}| \sim M$ at the beginning of the oscillations (if there was a previous period of inflation then the typical momentum would be much smaller). It is possible to estimate the annihilation rate from the results in [29] as $\Gamma_{2 \rightarrow 2} \sim M^{7} v^{2} / f^{8}$ at the first stages of oscillation. In the regions in which we will be interested, we find $\Gamma_{2 \rightarrow 2} \ll M$. In addition, those processes in which four zero-momentum branons are transformed into two branons with energies $E=2 M$ were already considered in the first reference in [32]. In this case Bose enhancement effects are important. The results obtained for axions can be immediately translated into the branon case, simply identifying $v$ with the Peccei-Quinn scale. It was shown that in the initial stages of oscillations, which corresponds to $\pi \sim v$, the decay rate was $\Gamma_{4 \pi \rightarrow 2 \pi} \sim M$. Again, this rate would be smaller if there was a period of inflation. Therefore the necessary condition to avoid the energy depletion of the branon condensate is $H\left(T_{R H}\right) \geq M$. Making use of the Friedmann equation in a radiation dominated universe, this implies $T_{R H} \gtrsim\left(M M_{P}\right)^{1 / 2}$, which automatically ensures $T_{R H} \gg \Lambda$. Since $\Gamma$ decreases very fast due to the universe expansion, it will never become comparable to $H$. Accordingly, the branon condensate energy density essentially is not reduced by particle production, but only diluted by the Hubble expansion. We see that the reheating temperature should satisfy the condition $T_{i} \simeq\left(M M_{P}\right)^{1 / 2}$

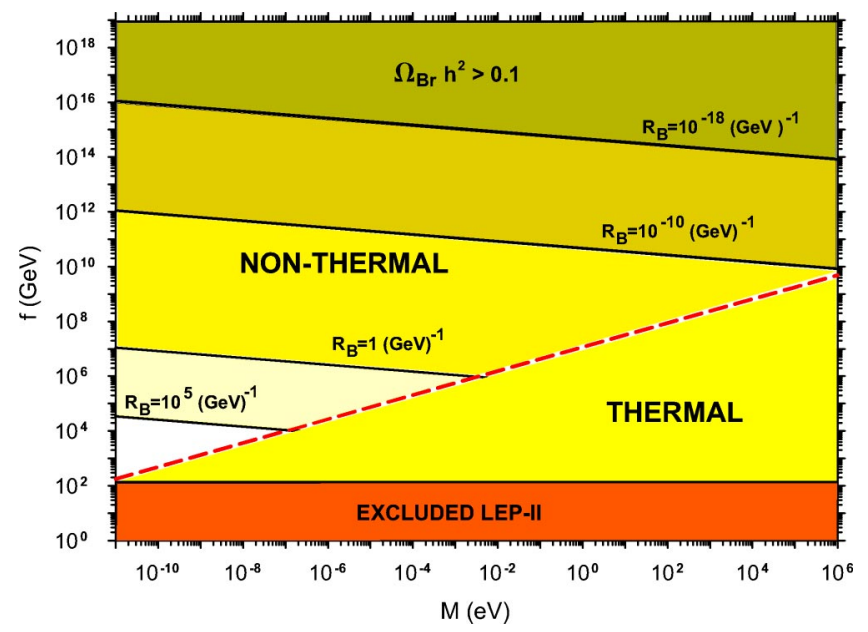

FIG. 2. Thermal vs non-thermal branon regions in the $f-M$ plane. The dashed (red) line separates the two regions and corresponds to $T_{i} \simeq T_{f}$. The continuous (black) lines correspond to $\Omega_{B r} h^{2} \simeq 0.1$ for different values of the radius of the extra dimensions $R_{B}=10^{5}, 1,10^{-10}, 10^{-18} \mathrm{GeV}^{-1}$.

$<T_{R H}<T_{f}$ in order for the condensate to form and survive until the present. Therefore if $T_{i}>T_{f}$ the above interval disappears and only thermal relics can be present. In the opposite case, $T_{i}<T_{f}$, we can also have non-thermal production. In the case of light branons, a good estimation for the freezeout temperature was obtained in $[23,29]$ : $\log \left(T_{f} / \mathrm{GeV}\right)$ $\simeq(8 / 7) \log (f / \mathrm{GeV})-3.2$. In Fig. 2 the $T_{i}=T_{f}$ curve which separates the thermal and non-thermal regions is plotted as a dashed line. We see that for sufficiently light or weakly coupled branons non-thermal production is possible.

Following the same steps as in the axionic case, we can calculate the energy density that is stored today in the form of branon oscillations. Assuming that $M$ does not depend on the temperature, it is given by [32]

$$
\Omega_{B r} h^{2} \simeq \frac{2.5 N v^{2} M T_{0}^{3}}{M_{P} T_{i} \rho_{0}} \simeq \frac{6.5 \times 10^{-20} N}{\mathrm{GeV}^{5 / 2}} f^{4} R_{B}^{2} M^{1 / 2}
$$

where $T_{0}$ and $\rho_{0}$ are the photon temperature and critical density today, respectively.

In particular it can be seen that within certain parameter regions, the above energy density can be cosmologically important. Thus in Fig. 2 we plot the $\Omega_{B r} h^{2}=0.1$ curves, corresponding to the current observational values, for different values of $R_{B}$ in the $f-M$ plane. Thus the region above each curve would be excluded by non-thermal branon overproduction. This is another manifestation of the cosmological moduli problem. Notice that in the allowed non-thermal regions of the figure the condition $f \ll M_{D}$ is also satisfied.

In the case in which the branon masses are larger than some SM particle mass, then the corresponding annihilation channel will open up and a detailed analysis of the energy loss rate will be necessary. In any case, the possibility of having heavier non-thermal branons is not excluded a priori. 


\section{CONCLUSIONS}

We have explored the different possibilities of branon dark matter in terms of the brane-world parameter $\left(N, M_{D}, f, M\right)$. We have shown that apart from the standard scenario [23] in which branons behave as collisionless thermal relics, the large parameter space of these models allows for branons to behave also as collisional or non-thermal dark matter candidates. In the collisional case, this opens an interesting possibility since the existing proposals for SDM candidates in the literature are very limited [21,33]. Neverthe- less, the allowed parameter region reduces only to warm collisional branons and therefore a detailed analysis of the actual effect on structure formation is needed.

\section{ACKNOWLEDGMENTS}

I would like to thank J. A. R. Cembranos, A. Dobado, and J. R. Peláez for useful comments and discussions, and also Alberto L. del Amo for additional motivation. This work was partially supported by the DGICYT (Spain) under the project numbers FPA 2000-0956 and BFM2002-01003.
[1] G. Jungman, M. Kamionkowski, and K. Griest, Phys. Rep. 267, 195 (1996).

[2] C. Muñoz, hep-ph/0309346.

[3] J. Navarro, C.S. Frenck, and S.D.M. White, Astrophys. J. 490, 493 (1997).

[4] B. Moore et al., Mon. Not. R. Astron. Soc. 310, 1147 (1999).

[5] W.J.G. de Blok and S.S. McGaugh, Mon. Not. R. Astron. Soc. 290, 533 (1997).

[6] B. Moore, Nature (London) 370, 629 (1994).

[7] R. Flores and J.R. Primack, Astrophys. J. Lett. 427, L1 (1994).

[8] A. Klypin et al., Astrophys. J. 522, 82 (1999).

[9] B. Moore et al., Astrophys. J. Lett. 524, L19 (1999).

[10] M. Kamionkowski and A.R. Liddle, Phys. Rev. Lett. 84, 4525 (2000).

[11] J.S. Bullock, A.V. Kravtsov, and D.H. Weinberg, Astrophys. J. 539, 517 (2000).

[12] J. Sommer-Larsen and A. Dolgov, Astrophys. J. 551, 608 (2001).

[13] D.N. Spergel and P.J. Steinhardt, Phys. Rev. Lett. 84, 3760 (2000).

[14] C.J. Hogan and J.J. Dalcanton, Phys. Rev. D 62, 063511 (2000)

[15] V.K. Narayanan, D.N. Spergel, R. Davé, and C.P. Ma, Astrophys. J. Lett. 543, L103 (2000).

[16] R. Davé, D.N. Spergel, P.J. Steinhardt, and B.D. Wandelet, Astrophys. J. 547, 574 (2001).

[17] O.Y. Gnedin and J.P. Ostriker, Astrophys. J. 561, 61 (2001).
[18] N. Yoshida, V. Springel, S.D.M. White, and G. Tormen, Astrophys. J. Lett. 544, L87 (2000).

[19] J. Miralda-Escudé, Astrophys. J. 564, 60 (2002).

[20] M. Markevitch et al., astro-ph/0309303.

[21] S. Hannestad and R.J. Scherrer, Phys. Rev. D 62, 043522 (2000).

[22] W.B. Lin et al., Phys. Rev. Lett. 86, 954 (2001).

[23] J.A.R. Cembranos, A. Dobado, and A.L. Maroto, Phys. Rev. Lett. 90, 241301 (2003).

[24] M. Bando et al., Phys. Rev. Lett. 83, 3601 (1999).

[25] R. Sundrum, Phys. Rev. D 59, 085009 (1999).

[26] A. Dobado and A.L. Maroto, Nucl. Phys. B592, 203 (2001).

[27] J.A.R. Cembranos, A. Dobado, and A.L. Maroto, Phys. Rev. D 65, 026005 (2002).

[28] J. Alcaraz, J.A.R. Cembranos, A. Dobado, and A.L. Maroto, Phys. Rev. D 67, 075010 (2003).

[29] J.A.R. Cembranos, A. Dobado, and A.L. Maroto, Phys. Rev. D 68, 103505 (2003).

[30] A. Dobado, A. Gómez-Nicola, A.L. Maroto, and J.R. Peláez, Effective Lagrangians for the Standard Model (SpringerVerlag, Berlin, 1997); J.F. Donoghue, E. Golowich, and B.R. Holstein, Dynamics of the Standard Model (Cambridge University Press, Cambridge, England, 1992).

[31] L. Hui, Phys. Rev. Lett. 86, 3467 (2001).

[32] J. Preskill, M.B. Wise, and F. Wilczek, Phys. Lett. 120B, 127 (1983); J.A. Frieman and A.H. Jaffe, Phys. Rev. D 45, 2674 (1992).

[33] A. Kusenko and P.J. Steinhardt, Phys. Rev. Lett. 87, 141301 (2001). 